\title{
Evaluation of a partial hospitalisation programme: good news and bad
}

\section{AIMS AND METHOD}

To evaluate a 50-place partial hospitalisation programme during its first year of operation. Data were recorded for consecutive referrals to the programme. Its effects on the admission unit were also assessed

\section{RESULTS}

The unit received over 200 referrals over 12 months, and more than $60 \%$ were for individuals who might otherwise have been admitted. The programme was associated with reductions in number and duration of hospital admissions and in bed occupancy rate; however, the proportion of urgent referrals to the programme doubled over the year, and after 12 months the occupancy rate was $96 \%$.

\author{
CLINICAL IMPLICATIONS \\ The programme was well received by \\ clients and well used by clinicians. \\ Although it initially reduced the \\ pressure on in-patient services, the \\ problems confronting the \\ programme at the end of the \\ evaluation mirrored those of \\ in-patient units.
}

The National Service Framework for Mental Health (Department of Health, 1999) and Mental Health Policy Implementation Guide (Department of Health, 2001) recommend the development of new treatment settings as an alternative to hospital admission. Evidence has accumulated over the past decade that the use of extended day hospital facilities and home treatment services can be an effective approach (Harrison et al, 1999). For example, a randomised controlled trial of day hospital $v$. in-patient care showed that up to $40 \%$ of those presenting for admission could be diverted to day care without any detrimental effect on their social or clinical outcome (Creed et al, 1990). Furthermore, day hospital treatment was less expensive than in-patient care (Creed et al, 1997).

In Newcastle upon Tyne, extremely high bed occupancy rates and increasing concerns over the quality of therapeutic work being undertaken in in-patient facilities led to the development of a partial hospitalisation programme. The first such programme opened in the West End of Newcastle and was the subject of a prospective evaluation of case mix, user satisfaction and impact on admissions.

\section{Overview of the service}

The West End of Newcastle has a population of just fewer than 150000 people. It is a deprived inner-city area with high levels of unemployment and a greater proportion of ethnic minority groups compared with the other catchment areas of the Newcastle Mental Health Care Trust. The core staff of the partial hospitalisation programme consisted of two occupational therapists (one basic grade, one senior II) and two nurses (one $\mathrm{G}$ grade, one $\mathrm{F}$ grade). Medical and other input was provided on a sessional basis from existing resources. Referrals to the programme were accepted from in-patient wards, community mental health teams and psychiatrists. The total case-load at any one time was up to 50 , although the frequency of attendance varied from an hour every 2 weeks, to every day.

The three broad aims of the partial hospitalisation programme were to provide:

- an alternative to in-patient care;

- transitional care for individuals who were about to be discharged from hospital;

- treatment and rehabilitation for individuals currently living in the community who had a history of repeated admissions for severe and enduring mental health problems.

Our hypothesis was that targeting these patient groups would reduce acute admissions by providing treatment in a 'least restrictive alternative' setting, would reduce lengths of stay by facilitating earlier discharge and would prevent repeated admissions by extending the support offered to vulnerable individuals in the community (Scott, 1995).

\section{Method}

Using a precoded pro-forma, data were recorded on consecutive referrals to the partial hospitalisation programme over the first year of its operation. The information included demographic details, DSM-IV diagnosis (American Psychiatric Association, 1994), past psychiatric history, and source of and reason for referral. Reasons for referral were: alternative to admission (ALTADM); facilitating the transition from hospital to home (TRANS); or increased community support for individuals with complex problems (SUPPORT). Prospective data recorded frequency of attendance at the PHP units and hospital admissions during the course of treatment under the programme.

To assess patient views of the service offered, current attenders $(n=47)$ were asked to complete the eight-item version of the Client Satisfaction Questionnaire (CSQ-8; Larson et al, 1979). Four additional openended questions were added, asking participants in what ways the programme had been helpful or unhelpful, what 
they liked or disliked about the service, and asking for any further comments or recommendations. The final score for the CSQ-8 is in the range 1-4 and is derived by dividing the total score by the number of items answered.

To explore any impact of the partial hospitalisation programme on the admission unit, information from the Patient Information Management System was used to assess measures of the unit's activity for the year prior to and the year after the introduction of the service. For each year we recorded the median number of occupied bed days per month, the mean bed occupancy rate per month, the mean number of admissions per month and the median length of stay per patient.

Data were analysed using the Statistical Package for the Social Sciences, version 9.5. Descriptive statistics were used to compare continuous and categorical ratings.

\section{Results}

\section{Referrals and activity}

The partial hospitalisation programme received 271 referrals in the first year of operation. Nearly three-quarters of them $(n=200)$ were from community staff and the rest were from hospital staff. Of the 271 referrals, 132 (63\%) were as an alternative to admission, $71(26 \%)$ were part of an early discharge programme and $68(21 \%)$ were referrals for additional community support. Fifty-three of the people referred did not attend for assessment, and a further nine subjects did not attend after their initial interview. From the minimal data-set available, these people did not appear to differ significantly in demographic characteristics from the 219 who did attend the programme.

The number of referrals to the programme per month ranged from 19 to 28 (mean 23.2, s.d. 4.1). Over the year, the proportion of referrals for urgent assessment increased significantly from $29 \%$ in months $1-3$ to $55 \%$ in months $10-12\left(\chi^{2}=7.1\right.$, d.f. $\left.=4 ; P=0.04\right)$. Of the 219 programme attenders, 162 were discharged over the year (turnover rate $77 \%$ ), leaving 47 of the available 50 places (96\%) occupied.

\section{Characteristics of programme attenders}

Details of the 209 people who attended the partial hospitalisation programme are given in Table 1. Their mean age was 42.2 years (s.d. 13.7, range 19-71) and they had a mean age of onset of mental disorder of 26.3 years (s.d. 10.1). Just under half (45\%) lived alone, and 58\% were female. Twenty-four were currently in paid employment. For 26 people (12\%), the programme was their first-ever contact with the mental health services. However, 109 people (52\%) had had at least one admission in the year prior to referral to the programme, of whom 23 had been admitted under a section of the Mental Health Act 1983. A third (33\%, n=69) met criteria for affective disorders (unipolar and bipolar), 31\% $(n=66)$ for schizophrenia or other psychoses, $17 \%(n=36)$ for other Axis I disorders (such as obsessive-compulsive disorder or eating disorder), 13\% $(n=28)$ for dual diagnosis (comorbid substance dependence and Axis I disorder) and $5 \%(n=10)$ had a primary diagnosis of personality disorder. Thirty-four individuals (16\%) required admission to hospital during the study period despite attending the programme.

There were relatively few differences between the three referral subgroups except that statistically significantly more individuals were referred as an alternative to admission ( $53 \% ; \chi^{2}=8.1$, d.f. $=4 ; P=0.03$ ) compared with those referred for other reasons. No person in the TRANS group was new to the service, compared with $16 \%$ of the ALTADM group ( $\chi^{2}=6.1$, d.f. $=4 ; P=0.05$ ), and significantly fewer people in the TRANS group (8\%) compared with the rest of the sample (19\%) required readmission while attending the programme $\left(\chi^{2}=6.3\right.$, d.f. $\left.=4 ; P=0.04\right)$.

\section{Client satisfaction}

Thirty of the 47 people currently treated by the programme (64\%) returned a completed CSQ-8 form. The overall scores ranged from 1.78 to 4.0 , with a mean of 3.1 indicating that the clients were mostly satisfied with the service. Thirteen clients gave an overall rating of 3 or above, while only three clients indicated indifference or mild dissatisfaction, with a CSQ score below 2 .

Answers to specific questions revealed that two-thirds of clients felt that the programme met most of their needs, but seven people felt that it was not appropriate and another three found the travelling a strain.

One in five of those who completed the CSQ commented that the atmosphere of partial hospitalisation programme felt safe and positive, and two in five reported that the staff were accessible and supportive. Nineteen per cent of respondents said that they liked the structure that the programme gave to their lives, while $73 \%$ commented that it offered them the opportunity to socialise with others. Twenty-seven per cent of respondents said that the programme had taught them new skills and problem-solving techniques, but eight others said they disliked it because they did not get on with some or all of the other clients. Eight respondents suggested improvements to the programme, such as extending the number of hours and also the overall length of time that clients could attend.

\section{Impact on the in-patient unit}

The year following the introduction of the partial hospitalisation programme was associated with reductions in several measures of admissions unit activity (Table 2). The median number of occupied bed days per month fell from 1461 (interquartile range (IQR) 1291-1506) to 1209 (IQR 1011-1336), and the mean bed occupancy rate per month fell by $18 \%$ (from $113.8 \%$ to $95.7 \%$ ). The mean number of admissions per month fell from 57.3 (s.d. 12.2) to 47.4 (s.d. 10.7) and the median length of stay per patient fell by $24 \%$ from 17 days (IQR $11-36$ ) to 13 days (IQR 6-27). The latter trend just failed to reach statistical significance (Mann-Whitney $U$ test, $P=0.06$ ). However, original papers 
original papers

$\operatorname{ALTADM}(n=110)$

TRANS $(n=53) \quad$ SUPPORT $(n=46) \quad$ Significance $(P)$

\begin{tabular}{|c|c|c|c|c|}
\hline Referrals to programme (\%) & 53 & 25 & 22 & 0.03 \\
\hline Age, years (mean (s.d.)) & $41.2(12.6)$ & $43.5(13.9)$ & $46.2(14.2)$ & 0.09 \\
\hline Age at onset of mental disorder, years (mean (s.d.)) & $25.3(10.2)$ & $26.2(9.8)$ & $27.9(9.3)$ & 0.2 \\
\hline Gender: female $(n(\%))$ & $67(61)$ & $34(64)$ & $21(47)$ & 0.1 \\
\hline Living alone $(n(\%))$ & $51(46)$ & $17(32)$ & $27(59)$ & 0.09 \\
\hline Currently employed ( $n(\%))$ & $13(12)$ & $7(13)$ & $4(9)$ & 0.5 \\
\hline Diagnosis $(n(\%))$ & & & & NS \\
\hline Affective disorder & $39(35)$ & $16(30)$ & $14(30)$ & \\
\hline Schizophrenia/other psychosis & $32(29)$ & $21(40)$ & $13(28)$ & \\
\hline Other Axis I disorder & $15(14)$ & $8(15)$ & $13(28)$ & \\
\hline Comorbid substance dependence and Axis I disorder & $19(17)$ & $7(13)$ & $2(4)$ & \\
\hline Personality disorder & $5(5)$ & $1(2)$ & $4(9)$ & \\
\hline Number of admissions in previous 12 months (mean (s.d.)) & $1.9(1.7)$ & $1.2(1.1)$ & $1.1(1.3)$ & 0.08 \\
\hline First mental health service contact $(n(\%))$ & $18(16)$ & 0 & $8(17)$ & 0.05 \\
\hline Admitted from programme $(n(\%))$ & $22(20)$ & $4(8)$ & $8(17)$ & 0.04 \\
\hline
\end{tabular}

ALTADM, referred as an alternative to admission; SUPPORT referral to increase support for individual in the community; TRANS, referral to facilitate transition from hospital to home

there was one statistically significant difference in admission patterns: in the year prior to the introduction of the programme, $44 \%$ of admissions were for 2 weeks or less and $18 \%$ for 3 months or more; in the year after the introduction of the programme the equivalent figures were $57 \%$ and $8 \%$ respectively $\left(\chi^{2}=6.2\right.$, d.f. $=4$; $P=0.05$ ).

\section{Discussion}

It is important to highlight two limitations of this study. First, this is an independent cohort study (without masking) of an evolving service, not a randomised controlled trial of a more established unit. Second, the hospital admissions data might have been influenced by many factors other than the introduction of the partial hospitalisation programme. Having noted these issues, this evaluation suggests that the programme can provide a 'least restrictive alternative' treatment setting for a significant proportion of individuals who need input from specialist mental health services. The service remained focused on its target population: $90 \%$ of those attending had severe mental disorders or complex, enduring problems, and over $50 \%$ had previously experienced hospital admission. There was evidence that the programme reduced the pressure on the in-patient unit: data for the year before and the year after the introduction of this programme demonstrated trends that reached statistical significance in some instances, for reductions in the number of occupied bed days, the mean bed occupancy rates, and the number of admissions and median lengths of stay.

These positive aspects of the partial hospitalisation programme are tempered by the emergence of three problems. First, over the course of the year, 30 individuals attending the programme (22 referred as an alternative to admission) were eventually admitted to hospital, suggesting that the programme can delay but not prevent admission in about $15 \%$ of referrals. Second, the small number of core staff and lack of dedicated medical input meant that it was not always possible to offer a place to individuals with the most acute severe problems; at the same time, staff making the referrals increasingly wanted the programme to be a substitute for in-patient care, and there was a significant increase in the number of urgent referrals (rising from $29 \%$ to $55 \%$ of all referrals). Last, the ability of the programme to

Table 2. In-patient unit activity in the year before and year after the introduction of the partial hospitalisation programme

\begin{tabular}{|c|c|c|c|}
\hline Activity & Year before & Year after & Significance $(P)$ \\
\hline Number of occupied bed days per month (median (25th-75th quartile)) & $\begin{array}{c}1461(1291- \\
1506)\end{array}$ & $1209(1011-1336)$ & 0.07 \\
\hline Bed occupancy rate per month (mean \% (s.d.)) & $113.8(14.3)$ & $95.7(16.7)$ & 0.09 \\
\hline Number of admissions per month (mean (s.d.)) & $57.3(12.2)$ & $47.4(10.7)$ & 0.07 \\
\hline Length of stay, days (median ( 25 th -75 th quartile)) & $17(11-36)$ & $13(6-27)$ & 0.06 \\
\hline Admitted for $<2$ weeks & 44 & 57 & \multirow{4}{*}{0.05} \\
\hline Admitted for $>2$ weeks, $<1$ month & 19 & 24 & \\
\hline Admitted for $1-3$ months & 17 & 11 & \\
\hline Admitted for $>3$ months & 18 & 8 & \\
\hline
\end{tabular}


offer transitional or time-limited community support was compromised by difficulties in finding alternative facilities for clients to move to after completing the programme; as a result, some individuals started to see the programme as a long-term day care facility. All of the issues described appeared to contribute to the reduced rate of turnover of clients at the programme's unit and to the occupancy rate (96\%); anecdotally, this appeared to influence the ability of the programme to deliver a range of therapies. Ironically, by the end of the evaluation period, the operational difficulties encountered by the programme were a mirror image of the problems noted in the in-patient unit that had prompted the development of the partial hospitalisation programme in the first place.

In summary, although the partial hospitalisation programme had clear goals for reducing acute admissions, preventing readmission of vulnerable clients and facilitating earlier discharge from in-patient care, this study suggests its early success may not be sustainable. The ability of the programme to continue to take urgent referrals may be compromised unless additional medical and non-medical staffing is provided to enable it to manage a greater proportion of severe acute cases. The programme's ability to offer time-limited treatment to vulnerable individuals may be undermined by the lack of alternative care for individuals to move on to (or back to) in the community. Finally, although the programme was initially devised to target three patient populations simultaneously, the increasing bias toward urgent referrals and the greater proportion of referrals for the partial hospitalisation programme as an alternative to in-patient care, potentially distorts the intervention programmes being offered. It may be that the unit will need to have a more selective focus in the future if it is to function effectively and data on the effectiveness of the partial hospitalisation programme is required.

\section{Acknowledgements}

Janine Williamson (formerly research assistant in Newcastle upon Tyne) collected data and wrote a preliminary draft of the report on service satisfaction for the Newcastle and North Tyneside District Health Authority. Unfortunately, after Janine left Newcastle to join a clinical psychology training course, we have not been able to reestablish contact to obtain her permission to include her as an author of this paper. However, we wish to acknowledge her considerable input to this project. The Newcastle and North Tyneside District Health Authority funded this project.

\section{References}

AMERICAN PSYCHIATRIC ASSOCIATION (1994) Diagnostic and Statistical Manual of Mental Disorders (4th edn) (DSM-IV). Washington, DC APA.

DEPARTMENT OF HEALTH (1999) The National Service Framework for Menta Health. London: HMSO

DEPARTMENT OF HEALTH (2001) The Mental Health Policy Implementation Guide London: HMSO.

CREED, F., BLACK, D., ANTHONY, P., et al (1990) Randomised controlled trial of day patient versus inpatient psychiatric treatment. BMJ, 300, 1033-1037.

and in patient psychiatric treatment. BMJ, 314, 1382-1385.

HARRISON, J., POYNTON, A MARSHALL, J., et al (1999) Open all hours: extending the role of the psychiatric day hospital. Psychiatric Bulletin, 23, 400-404.

LARSON, D., ATKINSON, O., HARGREAVES, W., et al (1979) Assessment of client satisfaction: development of a general scale. Evaluation Programme Planning, 2, 197-207.

SCOTT, J. (1995) A 12 month pilot evaluation of a British partia hospitalisation program. International CREED, F., MBAYA, P., LANCASHIRE, S., Journal of Mental Health, 24, 60-69. et al (1997) Cost effectiveness of day

* Mary Jane Tacchi Consultant Psychiatrist, Crisis Assessment and Treatment Service, Ravenswood Clinic, Ravenswood Road, Heaton, Newcastle uponTyne NE6 5TX, Suresh Joseph Consultant Psychiatrist, Newcastle General Hospital, Newcastle uponTyne, Jan Scott Professor of Psychological Treatments Research, Institute of Psychiatry, London original

papers 